\title{
The relation between consciousness and attention: An empirical study using the priming paradigm
}

\author{
Eva Van den Bussche ${ }^{\mathrm{a}, *, 1}$, Gethin Hughes ${ }^{\mathrm{b}}$, Nathalie Van Humbeeck ${ }^{\mathrm{a}}$, Bert Reynvoet ${ }^{\mathrm{a}}$ \\ a Department of Psychology, University of Leuven, Leuven, Belgium \\ ${ }^{\mathrm{b}}$ Laboratoire Psychologie de la Perception, Université Paris Descartes, Paris, France
}

\section{A R T I C L E I N F O}

\section{Article history:}

Received 28 May 2008

Available online 25 January 2010

\section{Keywords:}

Attention

Consciousness

Response priming

\begin{abstract}
A B S T R A C T
Dehaene, Changeux, Naccache, Sackur, and Sergent (2006) and Koch and Tsuchiya (2007) recently proposed taxonomies that distinguish between four processing states, based on bottom-up stimulus strength and top-down attentional amplification. The aim of the present study was to empirically test these processing states using the priming paradigm. Our results showed that attention (prime attended or not) and stimulus strength (prime presented subliminally or not) significantly modulated priming effects: either receiving top-down attention or possessing sufficient bottom-up strength was a prerequisite for a stimulus to elicit priming. When both top-down attention and sufficient bottom-up strength were present, the priming effect was boosted. The origins of the observed priming effects also varied between different processing states. We can conclude that our empirical study using the priming paradigm confirmed the presence of four processing states, which displayed a differential pattern of response priming effects and differential origins of the response priming effects.
\end{abstract}

(c) 2010 Elsevier Inc. All rights reserved.

\section{Introduction}

One way to enhance our understanding of the mechanisms underlying consciousness is to contrast conscious and non-conscious visual processing. Recently, theoretical taxonomies have been proposed to distinguish conscious and nonconscious processing states. Dehaene et al. (2006) for example proposed a taxonomy based on the global neuronal workspace hypothesis (Baars, 1989) that distinguishes between subliminal, preconscious and conscious processing. According to this global neuronal workspace theory (Dehaene \& Naccache, 2001), bottom-up activation based on sufficient stimulus strength is necessary, but not sufficient for conscious access. More specifically, two thresholds in human information processing are assumed: a minimal duration to reach neural activity, and the significantly longer duration to reach the consciousness threshold. Subliminally presented or masked stimuli, which reach the first threshold but not the second one, can cause a burst of weak and short-lived activity, but will not reach a conscious state. Thus, insufficient bottom-up activation will prevent conscious access, indicating that bottom-up activation of a stimulus is necessary for conscious access. However, even when sufficient bottom-up strength is present, this does not necessarily mean that the consciousness threshold will be crossed. For example, in a study of the attentional blink, Sergent, Baillet, and Dehaene (2005) showed that stimuli displaying a strong and continuing activation were accompanied by a complete lack of conscious report. Another example is inattentional blindness, where a fully-visible, but unexpected object is not noticed, because attention is engaged

\footnotetext{
* Corresponding author. Address: Faculty of Psychology and Educational Sciences, University of Leuven, Tiensestraat 102, 3000 Leuven, Belgium. Fax: +32 (0) 16326099 .

E-mail address: Eva.Vandenbussche@psy.kuleuven.be (E. V. d. Bussche).

${ }^{1}$ Postdoctoral researcher of the Research Foundation - Flanders, Belgium.
} 
on another task, event or object (e.g. Mack \& Rock, 1998). Thus, bottom-up activation is not always sufficient for conscious access. What is additionally needed for conscious perception according to the global neuronal workspace theory is top-down attentional amplification: a stimulus must not only reach sufficient bottom-up activation, this activity must also be amplified and maintained over a minimal duration to reach the consciousness threshold. The latter is achieved by top-down attentional amplification. This top-down amplification causes the stimulus-evoked activation to extend to highly interconnected associative areas, allowing information to be held on-line for a long duration and allowing information to spread rapidly to many brain systems. Dehaene and colleagues argue that these two properties are typical of conscious perception.

Thus, according to the global neuronal workspace theory, both sufficient bottom-up strength and top-down attentional amplification are needed for conscious perception. Based on this, Dehaene et al. (2006) proposed a taxonomy that distinguishes three types of processing: (1) subliminal processing, where information inaccessibility is caused by insufficient bottom-up activation. When this subliminal information does not receive top-down attention, the activation will remain very weak. However, when attentional amplification is oriented towards it, the processing of the subliminal stimulus can be strengthened, although conscious access will never be reached. This is in line with the finding that primes made invisible by metacontrast masking remained unseen, even when they received attention (Kentridge, Nijboer, \& Heywood, 2008); (2) preconscious processing, where information inaccessibility is caused by a lack of top-down attentional amplification. These stimuli do possess sufficient bottom-up strength, so they can potentially reach conscious access, but they are not consciously accessed yet, because they have not received attention yet, which is also reflected in an incapability to report these stimuli; (3) conscious processing, where the bottom-up stimulus strength is sufficient and attentional amplification is present, leading to conscious access. The former two states are both non-conscious, whereas the latter is a conscious state.

As Dehaene et al. (2006), Koch and Tsuchiya (2007) proposed a two-by-two taxonomy and argued that top-down attention and consciousness are two distinct phenomena that can be dissociated. More specifically, they distinguish four states. At the one end of the spectrum, there is "attention with consciousness", where subjects become conscious of objects or events that they attend to. At the other end, there is "no attention no consciousness", which comprises objects or events that do not benefit from a top-down attentional bias and therefore remain unreported. In between, the two most controversial quadrants are situated. At the one hand, "attention without consciousness" where subjects attend to a location, but still fail to see one or more attributes of an object at that location. Masked priming can for example be situated in this quadrant: attended masked stimuli are able to elicit priming effects. At the other hand, "consciousness in the near absence of attention" where even though no or very little attention is dedicated to a certain object or event, subjects are still able to partially report it. For example, it has been shown that subjects are able to distinguish between male and female faces in the (unattended) periphery (Reddy, Reddy, \& Koch, 2006). This taxonomy is in many aspects similar to the one proposed by Dehaene et al. (2006). However, unlike Dehaene et al., who state that in the preconscious condition, conscious reportability is not possible, these authors claim that conscious perception can occur without attention or at least in the near absence of attention.

The first aim of the present study was to empirically test these taxonomies and the distinct processing states using the priming paradigm. In the priming paradigm, the processing of a target stimulus is enhanced when it is preceded by a related prime stimulus, compared to when it is preceded by an unrelated prime stimulus. The priming effect is expressed by a faster and/or more accurate response to related prime-target pairs compared to unrelated pairs. In the present study, we will specifically examine response priming. For example, when subjects are asked to categorize target numbers between 1 and 9 as smaller or larger than 5 by pressing a left-hand button when the target is smaller than 5 and a right-hand button when it is larger than 5, they will respond faster to congruent trials (e.g. 1-4), where prime and target belong to the same semantic category and require the same response, compared to incongruent trials (e.g. 1-7), where prime and target belong to different semantic categories and require different responses (i.e. response congruency effect or RCE, e.g. Dehaene et al., 1998). We note that this kind of response priming has to be distinguished from true semantic priming, where priming effects are not confounded by response effects and stem solely from the semantic relatedness between prime and target. Using this paradigm and presenting primes and targets at two different places on the screen, we were able to manipulate both the bottom-up strength and the top-down attentional amplification of the prime stimuli. In line with the suggestions of Dehaene et al. (2006), the stimulus strength was manipulated by presenting the primes either subliminally (i.e. for a very short duration and masked) indicating that these primes had weak stimulus strength, or clearly visible (i.e. for a longer duration and unmasked) indicating that these primes had enough stimulus strength to reach the consciousness threshold. The topdown attentional amplification was manipulated by directing the subjects' attention either towards the primes or away from the primes. In our design, a cue was presented that always validly indicated at which of the two places the target would appear on each trial. Thus, attention was always drawn to the place where the target would appear. The primes could either be presented at the same location as the target, indicating that they also received top-down attention, or at a different location, indicating they did not receive attention. We note that manipulating spatial attention and using an exogenous cue is only one possible way to manipulate attention, and different approaches (e.g. an endogenous and/or a temporal manipulation) can be used. Using these two manipulations, we empirically recreated the four processing states mentioned in the taxonomies of Dehaene et al. (2006) and Koch and Tsuchiya (2007), and we can hypothesize in which conditions priming effects should be observed: (1) for subliminal primes that do not receive attention (i.e. weak bottom-up activation and no top-down attentional amplification), no or only a very small amount of priming is expected; (2) for subliminal primes that do receive attention (i.e. weak bottom-up activation but top-down attentional amplification), we also expect, in line with previous demonstrations (e.g. Fabre, Lemaire, \& Grainger, 2007; Lachter, Forster, \& Ruthruff, 2004; Van den Bussche \& Reynvoet, 2007), to observe limited but significant priming effects; (3) for clearly visible primes that do not receive attention 
(i.e. strong bottom-up activation but no top-down attentional amplification), we also expect to observe limited priming effects; (4) for clearly visible primes that also receive attention (i.e. strong bottom-up activation and top-down attentional amplification), we should obtain very strong priming effects. Thus, we hypothesize that attention will modulate the observed priming effects (receiving top-down attentional amplification will boost the observed priming effects), and stimulus strength will modulate the observed priming effects (possessing sufficient bottom-up strength will boost the observed priming effects).

The second aim of the present study was to ascertain how priming effects are caused in the four different conditions mentioned. It has, for example, been shown that when primes are made invisible by reducing their stimulus strength (e.g. by masking them), priming effects decayed when the prime-target SOA exceeded 200 ms (Kiefer \& Spitzer, 2000), whereas these effects persisted with SOAs above $500 \mathrm{~ms}$ when the primes were made invisible by directing attention away from them (e.g. by using an attentional blink paradigm) (Rolke, Heil, Streb, \& Henninghausen, 2001). Thus, these findings already indicate that the processing of the primes seems to differ in a condition where subliminal primes receive attention and in a condition where clearly visible primes do not receive attention. Finding qualitative differences between the mechanisms of the priming effects in the four conditions would provide further support for a distinction between the different processing states. More specifically, we will assess whether the observed priming effects are due to the fact that congruent trials are responded to faster (facilitative effect) or to the fact that incongruent trials are responded to slower (interference effect) or both and whether the cause of the priming effect differs between the different conditions.

\section{Method}

\subsection{Participants}

Fifty-two students participated in both parts of the experiment as partial fulfillment of a course requirement (45 females; mean age $=18$ ).

\subsection{Procedure}

As a first part of the experiment, all participants received the subliminal condition, where the prime had weak stimulus strength. Fig. 1 shows the sequence of a subliminal trial. First, a 480 ms mask existing of two hash marks (\#\#) was shown at both locations. Then, the cue (++) was presented for $120 \mathrm{~ms}$ at one location followed by a 27 ms mask (\#\#). Next, a prime appeared at one of the two locations for $27 \mathrm{~ms}$, while at the other location a meaningless filler symbol ( $\S$ ) was shown. They were replaced by an 80 ms mask (\#\#). Finally, the target was presented at the cued location until the participant responded. The inter-trial interval was $1000 \mathrm{~ms}$. All presentations were synchronized with the refresh cycle of the screen (13.3 ms). The cue always validly indicated the target location, but it validly indicated the prime on only half of the trials (i.e. attended). Immediately after completing the first part, the same 52 students also took part in the condition where primes were presented clearly visible and therefore possessed strong stimulus strength. Fig. 1 shows the sequence of a trial in this visible condition. First, a 480 ms mask (\#\#) was shown at both locations. Then, the cue (++) was presented for 120 ms at one

\section{Congruent trial}

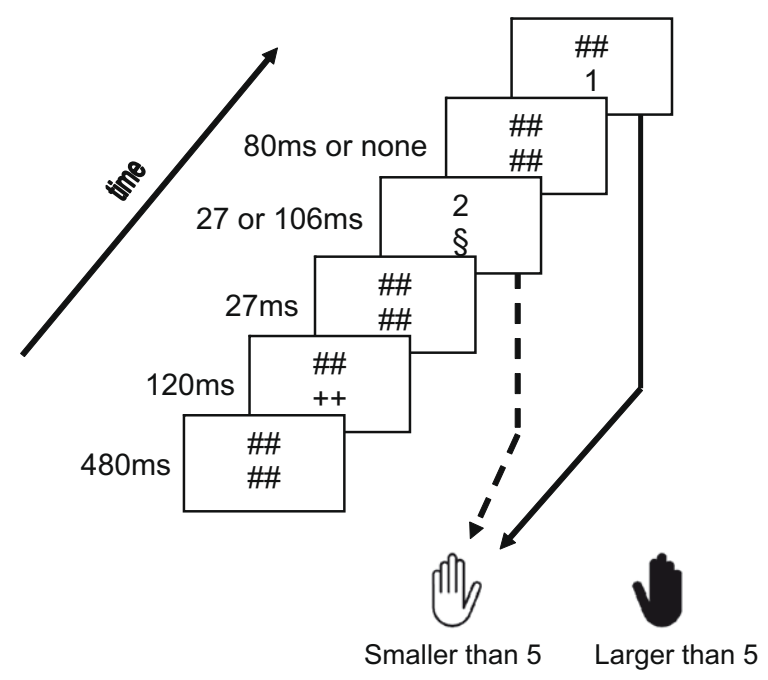

Fig. 1. Example of a congruent trial where the prime was unattended. In the subliminal condition, the prime was presented for 27 ms and the backward mask for $80 \mathrm{~ms}$. In the visible condition, the prime was presented for $106 \mathrm{~ms}$, and the backward mask was omitted. 
location followed by a 27 ms mask (\#\#). Next, a prime appeared at one of the two locations for $106 \mathrm{~ms}$, while at the other location the filler symbol $(\S)$ was shown. Finally, the target was presented at the cued location until the participant responded.

\subsection{Stimuli}

In both parts of the experiment, participants had to classify numbers as smaller or larger than 5 . Numbers 1, 4, 6 and 9 were used as targets and primes. Numbers $2,3,7$ and 8 were only presented as primes. In addition, the lowercase symbol " $\times$ " was used as a neutral prime. This led to 36 prime-target combinations ( 9 primes $\times 4$ targets). Stimuli were presented as white digits on a black background, ranged from $0.4 \mathrm{~cm}$ to $0.5 \mathrm{~cm}$ in width and were $0.7 \mathrm{~cm}$ high. Response assignment was varied across participants: half of them had to press 'a' when the target was smaller than 5 , and ' $p$ ' when it was larger than 5; the other half received the reversed response assignment. Participants were instructed to respond as quickly and accurately as possible. The stimulus locations were positioned $0.7 \mathrm{~cm}$ below and above the centre of the screen. Both prime and target could appear in one of these places, leading to four possible place combinations: two where the prime was attended (i.e. prime and target appeared at the same location) and two unattended (i.e. they appeared at different locations). In total, 144 combinations could be formed (36 prime-target combinations $\times 4$ place combinations). Sixty-four of these trials contained primes that were not presented as targets (i.e. novel primes), 64 contained primes that were also presented as targets (i.e. repeated primes), and 16 contained a neutral prime. Sixty-four trials were congruent, 64 were incongruent, and 16 were neutral. In each part of the experiment (subliminal and visible condition), the participants started with a 16trial training block presenting each target twice at each location, without a prime. The two experimental sessions contained two 144-trial blocks each.

\subsection{Post-tests}

After completing each part of the experiment, prime visibility was assessed using objective visibility tests. Participants were informed about the presence of the primes and were asked to participate in a post-test immediately after the subliminal condition and after the visible condition. In these 128-trial post-tests (excluding the neutral prime trials), using the same procedure as before, participants had to perform the same task but now on the prime instead of the target. If they were unable to categorize the prime, they had to guess. This post-test was used to calculate the prime visibility index for each condition.

\section{Results}

Wrong responses (on average 4.9\%) were discarded for RT analyses. We first report the results for the novel prime trials, since it has been argued that the influence of automatic stimulus-response (S-R) effects is reduced when using novel primes, and observed priming effects are therefore more likely to stem from semantic analysis of the primes (e.g. Damian, 2001). However, to ensure that the results for the repeated prime trials are nevertheless similar, we also report them in the second part. Third, all subjects received the same order of conditions (subliminal condition first, followed by the clearly visible condition). To ensure that training effects did not cause the observed pattern of results, we ran 18 additional subjects with the reversed order of conditions, and the results for this group are reported in the third part. Finally, the visibility analyses for the novel prime trials for the subjects who received the original order of conditions are reported in the fourth part in this section.

\subsection{Novel prime trials}

\subsubsection{Response priming}

To examine whether the RCEs were modulated by attention and stimulus strength, median RTs and mean error rates for novel prime trials were submitted to a repeated measures analysis with stimulus strength (weak or strong), cue validity (primes attended or not) and congruency (congruent or incongruent) as within-subject factors. Mean RTs and error rates as a function of these factors are listed in Table 1.

The repeated measures analysis performed on the RT data revealed the following results pattern. The main effect of congruency was significant $(F(1,51)=107.36, p<.001)$ with congruent trials on average responded to $18 \mathrm{~ms}$ faster than incongruent trials. The main effect of stimulus strength was also significant $(F(1,51)=29.70, p<.001)$ with trials with weak stimulus strength on average responded to $24 \mathrm{~ms}$ faster than trials with strong stimulus strength. However, these two factors significantly interacted with each other $(F(1,51)=35.28, p<.001)$ : the observed RCE was significantly larger in the strong stimulus strength condition $(31 \mathrm{~ms})$ than in the weak stimulus strength condition $(6 \mathrm{~ms})$, indicating that whether the primes possessed sufficient bottom-up stimulus strength or not significantly moderated response priming effects. The interaction between cue validity and congruency also reached significance $(F(1,51)=28.07, p<.001)$ : the observed RCE was significantly larger for the attended primes $(30 \mathrm{~ms})$ than for the unattended primes $(7 \mathrm{~ms})$, indicating that whether the primes were attended or not significantly moderated response priming effects. Finally, the three-way interaction between stimulus strength, cue validity and congruency was significant $(F(1,51)=15.54, p<.001)$, indicating a differential 


\section{Table 1}

Mean (SD) of the median RTs (in ms) and mean error rates (in \%) for the neutral, congruent and incongruent trials and the amount of RCE (incongruentcongruent), facilitation (neutral-congruent) and interference (incongruent-neutral) for the novel prime trials as a function of stimulus strength and cue validity.

\begin{tabular}{|c|c|c|c|c|c|c|c|}
\hline & & Neutral & Congruent & Incongruent & RCE & Facilitation & Interference \\
\hline \multirow[t]{4}{*}{ Weak } & Unattended & $495(47.0)$ & $493(46.0)$ & 495 (47.6) & 2 & 2 & 0.0 \\
\hline & & $4.0(5.1)$ & $3.5(3.5)$ & $4.0(4.4)$ & 0.5 & 0.5 & 0.0 \\
\hline & Attended & 498 (53.1) & 490 (49.5) & $500(44.3)$ & $10^{*}$ & $8^{*}$ & 2 \\
\hline & & $3.1(5.0)$ & $3.4(4.2)$ & $4.1(4.2)$ & 0.7 & -0.3 & 1.0 \\
\hline \multirow[t]{4}{*}{ Strong } & Unattended & $500(42.9)$ & $512(44.2)$ & $523(41.7)$ & $11^{* *}$ & $-12^{*}$ & $23^{* * * *}$ \\
\hline & & $4.7(7.1)$ & $5.0(5.3)$ & $5.0(5.1)$ & 0.0 & -0.3 & 0.3 \\
\hline & Attended & $517(48.6)$ & $495(48.4)$ & 545 (37.5) & $50^{* * * *}$ & $22^{* * * *}$ & $28^{* * *}$ \\
\hline & & $3.2(4.2)$ & $4.3(5.1)$ & $10.0(8.5)$ & $5.7^{* * *}$ & -1.1 & $6.8^{* * *}$ \\
\hline
\end{tabular}

${ }^{*} p<.05$.

${ }^{* * *} p<.01$.

**** $p<.001$

pattern of observed RCEs based on the stimulus strength and on the cue validity. Post hoc $t$-tests against the null mean indicated that the RCEs for weak attended primes $(10 \mathrm{~ms}, t(51)=2.59, p=.01)$, for strong unattended primes $(11 \mathrm{~ms}, t(51)=2.99$, $p=.004)$ and for strong attended primes (50 ms, $t(51)=10.16, p<.001)$ were significant. The RCE for the weak unattended primes was not significant $(2 \mathrm{~ms}, t(51)=0.68, p=.50)$.

The same repeated measures analysis performed on the error data revealed significant main effects of congruency $(F(1,51)=18.99, p<.001)$, stimulus strength $(F(1,51)=25.89, p<.001)$ and cue validity $(F(1,51)=6.87, p=.012)$. However, these factors all significantly interacted with each other. The interaction between stimulus strength and congruency $(F(1,51)=8.09, p=.006)$ indicated that the observed RCE was significantly larger in the strong stimulus strength condition (2.8\%) than in the weak stimulus strength condition (0.6\%). The interaction between cue validity and congruency also reached significance $(F(1,51)=12.58, p=.001)$ : the observed RCE was significantly larger for the attended primes $(3.2 \%)$ than for the unattended primes $(0.2 \%)$. The interaction between stimulus strength and cue validity was also significant $(F(1,51)=5.89, p=.019)$ : subjects made more errors on the strong conditions (5.0\% for unattended and $7.1 \%$ for attended primes) than on the weak conditions (3.7\% for both unattended and attended primes). Finally, the significant three-way interaction was again significant $(F(1,51)=17.75, p<.001)$. Post hoc $t$-tests against the null mean indicated that only the $\mathrm{RCE}$ for strong attended primes reached significance $(5.7 \%, t(51)=5.18, p<.001)$.

\subsubsection{Interference versus facilitation}

By including a neutral prime $(x)$ in the design, we were able to examine whether the RTs on congruent trials are being facilitated or whether the RTs on incongruent trials are being interfered. By subtracting the mean RT on the congruent trials from the mean $\mathrm{RT}$ on the neutral trials for each subject ( $\mathrm{RT}_{\text {neutral }}-\mathrm{RT}_{\text {congruent }}$ ), we can deduct how much the $\mathrm{RTs}$ are being facilitated relative to a neutral prime in each condition. By subtracting the mean RT on the neutral trials from the mean RT on the incongruent trials for each subject ( $\mathrm{RT}_{\text {incongruent }}-\mathrm{RT}_{\text {neutral }}$ ), we can deduct how much the $\mathrm{RTs}$ are being interfered relative to a neutral prime in each condition. Doing this, we can check how the RCEs we observed (see above) originated: either from a facilitative effect of the congruent trials, from an interference effect of the incongruent trials or a combination of both. Table 1 shows the amount of facilitation and interference of RTs as a function of stimulus strength and cue validity. We observed the following pattern. First, for weak unattended primes neither facilitation $(t(51)=0.70, p=.49)$ nor interference $(t(51)=-0.15, p=.88)$ of RTs emerges. Second, for weak attended primes only facilitation emerges $(t(51)=2.01, p=.049)$ and no interference $(t(51)=0.46, p=.65)$, indicating that the significant RCE we found in this condition was caused by a facilitative effect of the congruent trials. Third, for strong unattended primes we only observe interference $(t(51)=5.06$, $p<.001)$ and a reversed facilitation $(t(51)=-2.15, p=.036)$, which implies that the significant RCE we observed for this condition was caused by an interference effect of the congruent and incongruent trials. Fourth, for strong attended primes both facilitation $(t(51)=4.56, p<.001)$ and interference $(t(51)=5.46, p<.001)$ emerge, indicating that the significant RCE we found in this condition was caused by a combination of a facilitative effect of the congruent trials and an interference effect of the incongruent trials, which explains the magnitude of this observed RCE.

Analyses of variance also indicated that these observed facilitation and interference effects differed significantly between the four conditions (weak unattended, weak attended, strong unattended and strong attended) $(F(3,204)=9.29, p<.001$ and $F(3,204)=10.64, p<.001$ respectively).

The same analyses performed on the error data only revealed an interference effect for the strong attended primes $(t(51)=5.34, p<.001)$. None of the other facilitation and interference effects for the error rates were significant (see also Table 1). 


\subsection{Repeated prime trials}

The pattern of the results for the repeated primes was highly similar.

\subsubsection{Response priming}

Mean RTs and error rates for repeated prime trials as a function of stimulus strength, cue validity and congruency are listed in Table 2. The repeated measures analysis performed on the RT data revealed a main effect of congruency $(F(1,51)=100.82, p<.001)$ with congruent trials on average responded to $24.5 \mathrm{~ms}$ faster than incongruent trials. The main effect of stimulus strength was also significant $(F(1,51)=18.28, p<.001)$ with trials with weak stimulus strength on average responded to $17.5 \mathrm{~ms}$ faster than trials with strong stimulus strength. However, these two factors significantly interacted with each other $(F(1,51)=56.23, p<.001)$ : the observed RCE was significantly larger in the strong stimulus strength condition $(41 \mathrm{~ms})$ than in the weak stimulus strength condition $(8 \mathrm{~ms})$, indicating that whether the primes possessed sufficient bottom-up stimulus strength or not significantly moderated response priming effects. The interaction between cue validity and congruency also reached significance $(F(1,51)=16.20, p<.001)$ : the observed RCE was significantly larger for the attended primes ( $36 \mathrm{~ms}$ ) than for the unattended primes $(13 \mathrm{~ms})$, indicating that whether the primes were attended or not significantly moderated response priming effects. Furthermore, the interaction between cue validity and stimulus strength also reached significance $(F(1,51)=23.48, p<.001)$ : in the weak stimulus strength condition subjects responded faster to unattended primes $(6 \mathrm{~ms})$, whereas in the strong stimulus strength condition they responded faster to attended primes $(11 \mathrm{~ms})$. Finally, the three-way interaction between stimulus strength, cue validity and congruency was significant $(F(1,51)=8.59, p=.005)$, indicating a differential pattern of observed RCEs based on the stimulus strength and on the cue validity. Post hoc $t$-tests against the null mean indicated that the RCEs for weak attended primes $(13 \mathrm{~ms}, t(51)=2.78$, $p=.008)$, for strong unattended primes $(23 \mathrm{~ms}, t(51)=4.81, p<.001)$ and for strong attended primes $(59 \mathrm{~ms}, t(51)=9.46$, $p<.001)$ were significant. The RCE for the weak unattended primes was not significant $(3 \mathrm{~ms}, t(51)=0.82, p=.41)$.

The same repeated measures analysis performed on the error data revealed a main effect of congruency $(F(1,51)=17.23$, $p<.001$ ) with less errors being made on congruent compared to incongruent trials (3.9\% versus $6.1 \%$ ). The main effect of stimulus strength was also significant $(F(1,51)=25.47, p<.001)$ with less errors being made on trials with weak stimulus strength compared to trials with strong stimulus strength (4.0\% versus $6.1 \%$ ). The interaction between cue validity and congruency also reached significance $(F(1,51)=6.48, p=.014)$ : the observed RCE was significantly larger for the attended primes $(3.2 \%)$ than for the unattended primes (1.2\%). None of the other interactions were significant.

\subsubsection{Interference versus facilitation}

Table 2 shows the amount of facilitation and interference of RTs as a function of stimulus strength and cue validity. We observed the following pattern. First, for weak unattended primes neither facilitation $(t(51)=1.42, p=.16)$ nor interference $(t(51)=-0.88, p=.38)$ of RTs emerges. Second, for weak attended primes only facilitation emerges $(t(51)=2.24, p=.029)$ and no interference $(t(51)=1.04, p=.30)$, indicating that the significant RCE we found in this condition was caused by a facilitative effect of the congruent trials. Third, for strong unattended primes we only observe interference $(t(51)=5.04$, $p<.001)$ but no facilitation $(t(51)=-1.11, p=.27)$, which implies that the significant RCE we observed for this condition was caused by an interference effect of the incongruent trials. Fourth, for strong attended primes both facilitation $(t(51)=6.24, p<.001)$ and interference $(t(51)=3.30, p=.002)$ emerge, indicating that the significant RCE we found in this condition was caused by a combination of a facilitative effect of the congruent trials and an interference effect of the incongruent trials.

Table 2

Mean (SD) of the median RTs (in ms) and mean error rates (in \%) for the neutral, congruent and incongruent trials and the amount of RCE (incongruentcongruent), facilitation (neutral-congruent) and interference (incongruent-neutral) for the repeated prime trials as a function of stimulus strength and cue validity.

\begin{tabular}{|c|c|c|c|c|c|c|c|}
\hline & & Neutral & Congruent & Incongruent & RCE & Facilitation & Interference \\
\hline \multirow[t]{4}{*}{ Weak } & Unattended & 495 (47.0) & 489 (49.9) & $492(49.3)$ & 3 & 6 & -3 \\
\hline & & $4.0(5.1)$ & $3.7(4.2)$ & $4.4(5.6)$ & 0.7 & 0.3 & 0.4 \\
\hline & Attended & $498(53.1)$ & $490(55.4)$ & $503(42.7)$ & $13^{* *}$ & $8^{*}$ & 5 \\
\hline & & $3.1(5.0)$ & $2.6(3.9)$ & $5.2(6.2)$ & $2.6^{* *}$ & 0.5 & $2.1^{*}$ \\
\hline \multirow[t]{4}{*}{ Strong } & Unattended & $500(42.9)$ & $505(45.8)$ & $528(47.6)$ & $23^{* * *}$ & -5 & $28^{* * *}$ \\
\hline & & $4.7(7.1)$ & $4.7(5.2)$ & $6.3(6.6)$ & 1.6 & 0.0 & $1.6^{*}$ \\
\hline & Attended & $517(48.6)$ & $476(57.5)$ & $535(44.4)$ & $59^{* * *}$ & $41^{* * *}$ & $18^{* *}$ \\
\hline & & $3.2(4.2)$ & $4.8(4.9)$ & $8.6(7.4)$ & $3.8^{* * *}$ & $-1.6^{*}$ & $5.4^{* * *}$ \\
\hline
\end{tabular}

\footnotetext{
$p<.05$.

$p<.01$.

* $p<.001$.
} 
Analyses of variance also indicated that these observed facilitation and interference effects differed significantly between the four conditions (weak unattended, weak attended, strong unattended and strong attended) $(F(3,204)=16.04, p<.001$ and $F(3,204)=8.16, p<.001$ respectively).

The same analyses were performed on the error data (see also Table 2). We observed the following pattern. First, for weak unattended primes neither facilitation $(t(51)=0.41, p=.68)$ nor interference $(t(51)=0.76, p=.45)$ of RTs emerges. Second, for weak attended primes only interference emerges $(t(51)=2.60, p=.012)$ but no facilitation $(t(51)=0.70, p=.48)$, indicating that the significant RCE we found in this condition was caused by an interference effect of the incongruent trials. Third, for strong unattended primes we only observe a significant interference $(t(51)=2.05, p=.045)$ but no facilitation $(t(51)=-0.07, p=.95)$. Fourth, for strong attended primes interference $(t(51)=4.87, p<.001)$ and a reversed facilitation $(t(51)=-2.18, p=.03)$ emerge, indicating that the significant RCE we found in this condition was caused by an interference effect of the incongruent trials.

\subsection{Reversed order group}

All subjects received the same order of conditions: first the subliminal condition and then the clearly visible condition. The reason for this is that it is crucial that the subjects are unaware of the purpose of the study in the subliminal condition to enhance the subliminal nature of the primes. Furthermore, when novel primes are first shown clearly visible, we can question whether they can still be considered as "novel" in the subsequent subliminal condition. If not, then the observed priming effects are more likely to be due to S-R effects (see Damian, 2001) instead of semantic processing of the primes. Thus, the priming effects observed with the reversed order of conditions (clearly visible - subliminal) might have different underlying causes than the priming effects observed with the original order (subliminal - clearly visible). However, to ensure that training effects did not cause the observed pattern of results, we ran 18 additional subjects with the reversed order of conditions. A highly similar pattern of results was observed.

\subsubsection{Response priming}

Mean RTs and error rates for novel prime trials as a function of stimulus strength, cue validity and congruency are listed in Table 3. The repeated measures analysis performed on the RT data revealed a significant main effect of congruency $(F(1,17)=13.88, p=.002)$ with congruent trials on average responded to $17.5 \mathrm{~ms}$ faster than incongruent trials. The main effect of stimulus strength was also significant $(F(1,17)=72.38, p<.001)$ with trials with weak stimulus strength on average responded to $72 \mathrm{~ms}$ faster than trials with strong stimulus strength. However, these two factors significantly interacted with each other $(F(1,17)=8.75, p=.009)$ : the observed RCE was significantly larger in the strong stimulus strength condition (29.5 ms) than in the weak stimulus strength condition $(5.5 \mathrm{~ms})$, indicating that whether the primes possessed sufficient bottom-up stimulus strength or not significantly moderated response priming effects. The interaction between cue validity and congruency also reached significance $(F(1,17)=4.70, p=.04)$ : the observed RCE was significantly larger for the attended primes $(25 \mathrm{~ms})$ than for the unattended primes $(10 \mathrm{~ms})$, indicating that whether the primes were attended or not significantly moderated response priming effects. Furthermore, the interaction between cue validity and stimulus strength also reached significance $(F(1,17)=18.72, p<.001)$ : in the weak stimulus strength condition subjects responded faster to unattended primes $(6.5 \mathrm{~ms})$, whereas in the strong stimulus strength condition they responded faster to attended primes $(21.5 \mathrm{~ms})$. Finally, the three-way interaction between stimulus strength, cue validity and congruency was not significant $(F<1)$.

The same repeated measures analysis performed on the error data did not revealed any significant effects.

Table 3

Mean (SD) of the median RTs (in ms) and mean error rates (in \%) for the neutral, congruent and incongruent trials and the amount of RCE (incongruentcongruent), facilitation (neutral-congruent) and interference (incongruent-neutral) for the novel prime trials as a function of stimulus strength and cue validity for the subjects who received the reversed order of conditions (clearly visible - subliminal).

\begin{tabular}{|c|c|c|c|c|c|c|c|}
\hline & & Neutral & Congruent & Incongruent & RCE & Facilitation & Interference \\
\hline \multirow[t]{4}{*}{ Weak } & Unattended & 496 (59.3) & $493(35.3)$ & $491(29.4)$ & -2 & 3 & -5 \\
\hline & & $2.4(3.1)$ & $2.6(1.9)$ & $3.5(4.8)$ & 0.9 & -0.2 & 1.1 \\
\hline & Attended & $510(53.8)$ & 492 (33.5) & $505(36.2)$ & $13^{*}$ & $18^{*}$ & -5 \\
\hline & & $3.1(4.9)$ & $1.6(1.6)$ & $2.8(2.6)$ & 1.2 & 1.5 & -0.3 \\
\hline \multirow[t]{4}{*}{ Strong } & Unattended & $549(40.5)$ & $567(51.6)$ & $589(51.2)$ & $22^{*}$ & $-18^{*}$ & $40^{* *}$ \\
\hline & & $2.8(4.4)$ & $2.3(2.6)$ & $4.0(3.8)$ & 1.7 & 0.5 & 1.2 \\
\hline & Attended & $550(46.5)$ & $538(47.3)$ & 575 (39.0) & $37^{* * *}$ & 12 & $25^{* *}$ \\
\hline & & $3.8(3.8)$ & $1.9(2.2)$ & $2.1(2.4)$ & 0.2 & 1.9 & -1.7 \\
\hline
\end{tabular}

\footnotetext{
${ }^{*} p<.05$.

** $p<.01$.

*** $p<.001$.
} 


\subsubsection{Interference versus facilitation}

Table 3 shows the amount of facilitation and interference of RTs as a function of stimulus strength and cue validity. We observed the following pattern. First, for weak unattended primes neither facilitation $(t(17)=0.22, p=.83)$ nor interference $(t(17)=-0.38, p=.70)$ of RTs emerges. Second, for weak attended primes only facilitation emerges $(t(17)=2.09, p=.05)$ and no interference $(t(17)=-0.63, p=.54)$, indicating that the significant RCE we found in this condition was caused by a facilitative effect of the congruent trials. Third, for strong unattended primes we observe interference $(t(17)=4.13$, $p=.001)$ and a reversed facilitation $(t(17)=-2.65, p=.02)$, which implies that the significant RCE we observed for this condition was caused by an interference effect of the congruent and incongruent trials. Fourth, for strong attended primes interference $(t(17)=3.35, p=.004)$ emerges, but although the facilitation was in the expected direction, it did not reach significance $(t(17)=1.32, p=.20)$, indicating that the significant RCE we found in this condition was caused mostly by an interference effect of the incongruent trials.

Analyses of variance also indicated that these observed facilitation and interference effects differed (marginally) significantly between the four conditions (weak unattended, weak attended, strong unattended and strong attended) $(F(3,68)=2.51, p=.066$ and $F(3,68)=5.43, p=.002$ respectively $)$.

Since no significant response priming effects were found in the error data, it was not useful to examine the facilitation and interference effects here (see also Table 3 ).

\subsection{Prime visibility}

The prime visibility analyses were conducted on the subjects who received the original order and only on the novel prime trials, for reasons explained above. For the weak stimulus strength condition, participants could only correctly classify $53 \%$ of the unattended primes and $53 \%$ of the attended primes, even though they were fully informed about the presence of the primes in the post-tests. A direct measure of prime visibility was calculated for each subject in the form of standard $d^{\prime}$ measures. The measures are obtained by treating one level of the response category (i.e. smaller than 5) as signal and the other level (i.e. larger than 5) as noise. We obtained mean $d^{\prime}$ values of 0.17 for the unattended and 0.17 for the attended primes. Prime awareness did not significantly differ for the attended and the unattended primes $(t(51)=0.03, p=.97)$. $t$-tests against the null mean indicated the mean observed $d^{\prime}$ values were significantly different from 0 (respectively $t(51)=2.56$, $p=.01 ; t(51)=2.93, p=.005$ ). This indicates that primes could be classified slightly above chance. To further evaluate whether this above chance amount of prime visibility could account for the observed priming effect, Greenwald's analysis (e.g. Greenwald, Draine, \& Abrams, 1996) was performed. Using linear regression, non-significant correlations were found between the $d^{\prime}$ measures and the respective amounts of priming $(r=.05, F(1,50)=0.15, p=.70$ for the unattended primes and $r=.11, F(1,50)=0.59, p=.44$ for the attended primes). Crucially, the regression analysis showed that the priming index at the $d^{\prime}=0$ intercept was significant for the attended primes (intercept $=9 \mathrm{~ms} ; t(51)=2.09, p=.04$ ), but not for the unattended primes (intercept $=2 \mathrm{~ms} ; t(51)=0.50, p=.62$ ), demonstrating significant priming in the absence of prime perceptibility in the attended condition.

For the strong stimulus strength condition, subjects could classify on average $65 \%$ of the unattended primes and $82 \%$ of the attended primes. We obtained mean $d^{\prime}$ values of 0.78 for the unattended and 1.93 for the attended primes. As expected, prime awareness was significantly higher in the attended compared to the unattended condition $(t(51)=9.36, p<.001)$. Prime awareness was significantly above chance level for both the unattended and the attended primes (respectively $t(51)=8.02, p<.001 ; t(51)=16.79, p<.001)$.

To illustrate these prime visibility analyses, we plotted the observed response congruency effects (Figs. 2 and 3 ) against the corresponding $d^{\prime}$ values for each subject for the weak (Fig. 2) and strong (Fig. 3) stimulus strength conditions separately. Then, regression lines were fitted on these scatter clouds for the attended and unattended primes separately.

Finally, to ensure that the results did not originate from the subjects with higher $d^{\prime}$ values for the weak stimulus conditions, all analyses were conducted only on the 26 subjects with the lowest average $d^{\prime}$ values for the weak stimulus conditions (mean $d^{\prime}=-0.02$, range -0.43 to 0.16 ). The pattern of results was almost identical. Most importantly, the interaction between cue validity and congruency $(F(1,25)=11.41, p=.002)$ and the interaction between stimulus strength and congruency $(F(1,25)=11.47, p=.002)$ were also significant for the low $d^{\prime}$ group.

\section{Discussion}

The aim of the present study was twofold. First, we wanted to empirically test the distinct processing states as defined theoretically in the taxonomies proposed for example by Dehaene et al. (2006) and Koch and Tsuchiya (2007) using the priming paradigm. By manipulating bottom-up stimulus strength and top-down attention, we reconstructed the different processing states and we were able to examine in which conditions priming effects would emerge. Second, our study wanted to unravel the cause of the observed priming effects by investigating whether they were due to facilitation, interference or both, and whether the origin of the priming effects differed between the different processing conditions.

Our results showed that no significant priming was obtained for stimuli with insufficient bottom-up strength that did not receive top-down attentional amplification (weak unattended condition). We did obtain significant priming for stimuli with insufficient bottom-up strength that did receive top-down attentional amplification (weak attended condition) and for 


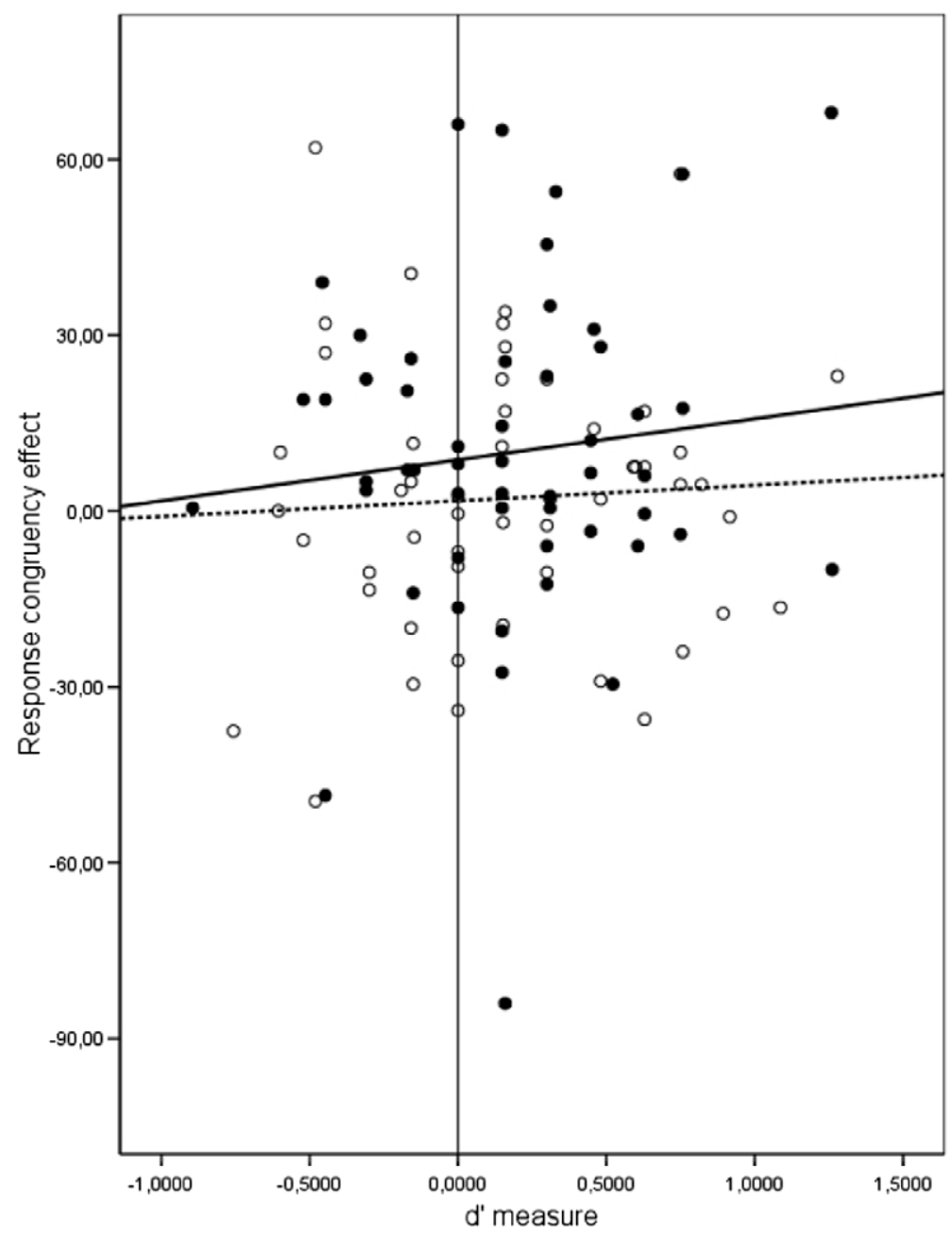

attended primes

unattended primes

attended primes

unattended primes

Fig. 2. The observed RCEs for the weak attended and unattended primes were plotted against the corresponding $d^{\prime}$ values for each subject. Regression lines were fitted for the attended and unattended primes separately.

stimuli with sufficient bottom-up strength that did not receive top-down attentional amplification (strong unattended condition). Logically, the strongest priming effects were obtained for stimuli with sufficient bottom-up strength that also received top-down attentional amplification (strong attended condition). Attention (whether the prime was attended or not) and stimulus strength (whether the prime was presented subliminally or clearly visible) both significantly modulated the observed priming effects: receiving top-down attention or possessing sufficient bottom-up strength boosted the observed priming effects. Furthermore, either receiving top-down attention or possessing sufficient bottom-up strength was sufficient to elicit significant priming, but when both were present, priming massively increased. These findings are completely in line with the processing states based on the theoretical taxonomies.

However, a few discrepancies between our empirical data and the theoretical predictions from the taxonomies should be mentioned. First, the key idea in the taxonomy of Dehaene et al. (2006) is that within the non-conscious states, it makes a major difference whether stimulus imperceptibility is achieved by a limitation in bottom-up strength (subliminal processing) or a lack of top-down attention (preconscious processing). Our results showed that in the situation where the stimulus has insufficient strength but receives attention (weak attended condition) the observed priming effect is caused by a facilitative effect of the congruent trials. Contrarily, when the stimulus has sufficient strength but does not receive attention (strong unattended condition), the observed priming effect is caused by an interference effect of the congruent and incongruent trials. This observation seems to imply that even though the amount of priming observed for weak attended primes and for strong unattended primes proved to be very similar, differential mechanisms lie at the basis of the priming observed for these two processing states, which would not only justify but indicate the necessity of distinguishing subliminal and preconscious processing, as Dehaene et al. (2006) did in their taxonomy. However, in the taxonomy of Dehaene et al., no reportability is assumed in the preconscious state, whereas we observed that the strong unattended primes in the response priming analysis were identified by the subjects above chance level. A possible reason for this might be that the way we 


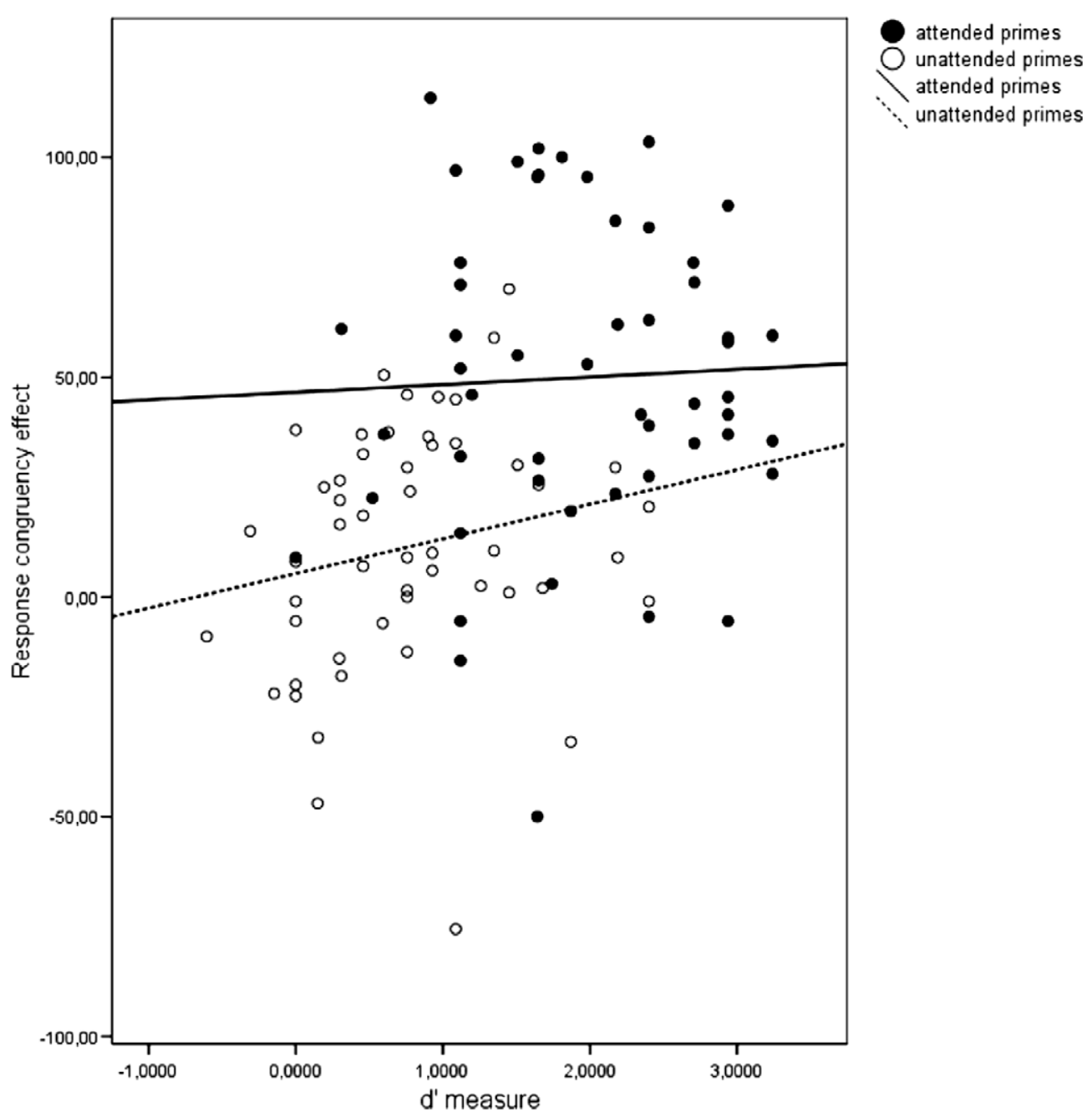

Fig. 3. The observed RCEs for the strong attended and unattended primes were plotted against the corresponding $d^{\prime}$ values for each subject. Regression lines were fitted for the attended and unattended primes separately.

assessed prime visibility was distinctively different from the paradigms, such as the attentional blink paradigm, used by Dehaene et al. to describe preconscious processing. Kouider, Dehaene, Jobert, and Le Bihan (2006) used a similar paradigm as ours and reported a similar observation in their preconscious condition: “... during debriefing, most participants claimed that they were aware that some flashed letters preceded the target stimuli, but they also claimed that they could hardly identify the primes or its relation to the target because they were busy performing the semantic decision task on the target stimuli. It is only during the subsequent forced-choice prime identification task, which required focused attention on the prime, that they could identify the prime stimuli" (p. 2027). Indeed, in our post-test subjects were also asked to attend to the primes and ignore the targets. Thus, it seems reasonable to suspect that the above chance visibility for strong unattended primes in the post-test was at least partially caused by attentional amplification towards the prime which was not present in the actual priming task. Dehaene (2008) recently argued that priority should be given to single-trial subjective reports in defining what constitutes a "conscious" trial (see also Lau, in press). According to him, unconscious processing can be inferred when objective performance is dissociated from subjective reports. Thus, in future studies, we should focus on designing an experiment where subjects have to perform a task on the target and rate their awareness of the prime on each single trial. Furthermore, the results of our visibility test clearly indicate that performance on the primes (as indicated by the post-tests) was significantly reduced in the strong unattended condition compared to the strong attended condition, indicating that attention was successfully manipulated in our design to some extent. Nevertheless, based on the above chance reportability in our strong unattended condition, we should be careful to label this condition as a good operationalization of Dehaene et al.'s (2006) preconscious condition. Rather, we should step away from their original taxonomy and label our strong unattended condition as a strong "less attended" condition. This is in line with the taxonomy of Koch and Tsuchiya (2007) where, instead of a preconscious processing state where no reportability is possible, a state of 
"consciousness in the near absence of attention" is defined. In this state, conscious perception can occur without attention according to Koch and Tsuchiya (2007). They base this claim on dual-task experiments where subjects are able to confidently perform certain discriminations on a peripheral (and thus unattended or less attended) target (e.g. Braun \& Julesz, 1998; Reddy et al., 2006). Although the authors mention that these studies do not rule out that some top-down attention is still being directed to the peripheral stimuli, they claim that their data indicate that conscious perception and reportability is possible in the "near absence of attention". Thus, based on this account, the above chance visibility for strong unattended primes we observed might have been caused by the fact that subjects are able to (to some extent) consciously report/ discriminate unattended stimuli.

Second, an unexpected observation arose in the strong unattended condition: both congruent and incongruent trials were responded to slower than neutral prime trials, although this interference was more prominent for the incongruent trials. This unexpected observation is similar to what has been observed in some Stroop and flanker tasks, where clearly visible distracters are shown and attention needs to be diverted from the distracter, because it is not required for the response, leading to similar conditions as in our strong unattended condition. For instance, Fias, Reynvoet, and Brysbaert (2001) found in this context that neutral trials were responded to faster than congruent and incongruent trials. Apparently, the presence of a task-relevant prime at an unattended location always leads to interference. An explanation for this observation could be that subjects were distracted by the task-relevant primes, which causes the target to be processed slower in the incongruent and congruent conditions than in the neutral prime condition. If this is indeed the case, one could argue that the unattended primes were not truly unattended. This would again lead us to labeling the strong unattended condition as a strong "less attended" condition, since our pattern of results clearly indicates that even though the strong unattended primes might have attracted some attention, it was still less than in the attended conditions. Regardless, results regarding facilitation and interference need to be interpreted with caution because the facilitative and interference effects are critically dependent on the neutral prime $(x)$ that we used. Finding the right neutral prime is difficult, and several authors already pointed out that the amount of facilitation and interference in Stroop and priming tasks might be determined by the choice of the control condition (Macleod, 1991; Neely, 1991).

Third, we should note that our results should be viewed in light of the specific task we used and the specific attentional mechanism we studied. Fabre et al. (2007) addressed this as a possible explanation for the fact that they did not observe attentional modulation of repetition priming, while others did (e.g. Besner, Risko, \& Sklair, 2005; Lachter et al., 2004; Marzouki, Grainger, \& Theeuwes, 2007): most of these studies used an exogenous manipulation of attention and examined spatial attention, whereas Fabre et al. used an endogenous manipulation of attention and examined temporal attention. Based on this, it could well be that if we, for example, studied temporal instead of spatial and endogenous instead of exogenous attention, our pattern of results would have been different, and so would have been the theoretical implications. Thus, when and how attention modulates subliminal priming might depend on the attentional mechanism under investigation and on the task and stimulus material being used, which clearly warrants more attention in future research.

We can conclude that our empirical study using the priming paradigm confirmed the presence of four processing states (subliminal unattended processing, subliminal attended processing, "less attended" processing and conscious processing), which displayed a differential pattern of response priming effects and differential origins of the response priming effects. Attention and stimulus strength modulated response priming: either receiving top-down attention or possessing sufficient bottom-up strength was necessary for a stimulus to elicit priming.

\section{References}

Baars, B. J. (1989). A cognitive theory of consciousness. Cambridge, MA: Cambridge University Press.

Besner, D., Risko, E. F., \& Sklair, N. (2005). Spatial attention as a necessary preliminary to early processes in reading. Canadian Journal of Experimental Psychology, 59, 99-108.

Braun, J., \& Julesz, B. (1998). Withdrawing attention at little or no cost: Detection and discrimination tasks. Perception Es Psychophysics, 60, 1-23.

Damian, M. F. (2001). Congruity effects evoked by subliminally presented primes: Automaticity rather than semantic processing. Journal of Experimental Psychology: Human Perception and Performance, 27, 154-165.

Dehaene, S. (2008). Conscious and nonconscious processes. Distinct forms of evidence accumulation? In C. Engel \& W. Singer (Eds.), The Strüngmann forum report. Better than conscious? Decision making, the human mind, and implications for institutions (pp. 22-49). MIT Press.

Dehaene, S., Changeux, J. P., Naccache, L., Sackur, J., \& Sergent, C. (2006). Conscious, preconscious, and subliminal processing: A testable taxonomy. Trends in Cognitive Sciences, 10, 204-211.

Dehaene, S., \& Naccache, L. (2001). Towards a cognitive neuroscience of consciousness: Basic evidence and a workspace framework. Cognition, 79, 1-37.

Dehaene, S., Naccache, L., Le Clec'H, G., Koechlin, E., Mueller, M., Dehaene-Lambertz, G., et al (1998). Imaging unconscious semantic priming. Nature, 395, $597-600$.

Fabre, L., Lemaire, P., \& Grainger, J. (2007). Attentional modulation of masked repetition priming and categorical priming in young and older adults. Cognition, 105, 513-532.

Fias, W., Reynvoet, B., \& Brysbaert, M. (2001). Are Arabic number processed as pictures in a Stroop interference task? Psychological Research, 65, $242-249$. Greenwald, A. G., Draine, S. C., \& Abrams, R. L. (1996). Three cognitive markers of unconscious semantic activation. Science, $273,1699-1702$.

Kentridge, R. W., Nijboer, T. C. W., \& Heywood, C. A. (2008). Attended but unseen: Visual attention is not sufficient for visual awareness. Neuropsychologia, 46, 864-869.

Kiefer, M., \& Spitzer, M. (2000). Time course of conscious and unconscious semantic brain activation. Neuroreport, 11, 2401-2407.

Koch, C., \& Tsuchiya, N. (2007). Attention and consciousness: Two distinct brain processes. Trends in Cognitive Sciences, 11, 16-22.

Kouider, S., Dehaene, S., Jobert, A., \& Le Bihan, D. (2006). Cerebral bases of subliminal and supraliminal priming during reading. Cerebral Cortex, 17, 2019-2029.

Lachter, J., Forster, K. I., \& Ruthruff, E. (2004). Forty-five years after Broadbent (1958): Still no identification without attention. Psychological Review, 111, $880-913$.

Lau, H.C. (in press). Volition and the functions of consciousness. In M. Gazzaniga (Ed.), Cognitive neurosciences IV. New York: MIT Press. 
Mack, A., \& Rock, I. (1998). Inattentional blindness. Cambridge, MA: MIT Press.

MacLeod, C. M. (1991). Half a century of research on the Stroop effect: An integrative review. Psychological Bulletin, 109, $163-203$.

Marzouki, Y., Grainger, J., \& Theeuwes, J. (2007). Exogenous spatial cueing modulates subliminal masked priming. Acta Psychologica, 126, 34-45.

Neely, J. H. (1991). Semantic priming effects in visual word recognition: A selective review of current findings and theories. In D. Besner \& G. Humphreys (Eds.), Basic processes in reading: Visual word recognition (pp. 264-336). Hillsdale, NJ: Lawrence Erlbaum.

Reddy, L., Reddy, L., \& Koch, C. (2006). Face identification in the near-absence of focal attention. Vision Research, 46, $2336-2343$.

Rolke, B., Heil, M., Streb, J., \& Henninghausen, E. (2001). Missed prime words within the attentional blink evoke an N400 semantic priming effect. Psychophysiology, 38, 165-174.

Sergent, C., Baillet, S., \& Dehaene, S. (2005). Timing of the brain events underlying access to consciousness during the attentional blink. Nature Neuroscience, $8,1391-1400$.

Van den Bussche, E., \& Reynvoet, B. (2007). Masked priming effects in semantic categorization are independent of category size. Experimental Psychology, 54, 225-235. 